\title{
Spin-glass phase transition and behavior of nonlinear susceptibility in the Sherrington-Kirkpatrick model with random fields
}

\author{
C. V. Morais, ${ }^{1,}{ }^{*}$ F. M. Zimmer, ${ }^{2}$ M. J. Lazo, ${ }^{3}$ S. G. Magalhães,${ }^{4}$ and F. D. Nobre ${ }^{5}$ \\ ${ }^{1}$ Instituto de Física e Matemática, Universidade Federal de Pelotas, 96010-900 Pelotas, Rio Grande do Sul, Brazil \\ ${ }^{2}$ Departamento de Fisica, Universidade Federal de Santa Maria, 97105-900 Santa Maria, Rio Grande do Sul, Brazil \\ ${ }^{3}$ Programa de Pós-Graduação em Física, Instituto de Matemática, Estatística e Física, Universidade Federal do Rio Grande, 96.201-900, \\ Rio Grande, Rio Grande do Sul, Brazil \\ ${ }^{4}$ Instituto de Fisica, Universidade Federal do Rio Grande do Sul, 91501-970 Porto Alegre, Rio Grande do Sul, Brazil \\ ${ }^{5}$ Centro Brasileiro de Pesquisas Físicas and National Institute of Science and Technology for Complex Systems, Rua Xavier Sigaud 150, \\ 22290-180, Rio de Janeiro, Rio de Janeiro, Brazil
}

(Received 28 April 2016; revised manuscript received 2 June 2016; published 17 June 2016)

\begin{abstract}
The behavior of the nonlinear susceptibility $\chi_{3}$ and its relation to the spin-glass transition temperature $T_{f}$ in the presence of random fields are investigated. To accomplish this task, the Sherrington-Kirkpatrick model is studied through the replica formalism, within a one-step replica-symmetry-breaking procedure. In addition, the dependence of the Almeida-Thouless eigenvalue $\lambda_{\mathrm{AT}}$ (replicon) on the random fields is analyzed. Particularly, in the absence of random fields, the temperature $T_{f}$ can be traced by a divergence in the spin-glass susceptibility $\chi_{\mathrm{SG}}$, which presents a term inversely proportional to the replicon $\lambda_{\mathrm{AT}}$. As a result of a relation between $\chi_{\mathrm{SG}}$ and $\chi_{3}$, the latter also presents a divergence at $T_{f}$, which comes as a direct consequence of $\lambda_{\mathrm{AT}}=0$ at $T_{f}$. However, our results show that, in the presence of random fields, $\chi_{3}$ presents a rounded maximum at a temperature $T^{*}$ which does not coincide with the spin-glass transition temperature $T_{f}$ (i.e., $T^{*}>T_{f}$ for a given applied random field). Thus, the maximum value of $\chi_{3}$ at $T^{*}$ reflects the effects of the random fields in the paramagnetic phase instead of the nontrivial ergodicity breaking associated with the spin-glass phase transition. It is also shown that $\chi_{3}$ still maintains a dependence on the replicon $\lambda_{\mathrm{AT}}$, although in a more complicated way as compared with the case without random fields. These results are discussed in view of recent observations in the $\mathrm{LiHo}_{x} \mathrm{Y}_{1-x} \mathrm{~F}_{4}$ compound.
\end{abstract}

DOI: 10.1103/PhysRevB.93.224206

\section{INTRODUCTION}

The presence of disorder in spin systems represents a permanent source of challenging problems, due to the richness of physical properties that emerge from the interplay between disorder and many-spin interactions. Random-field (RF) and spin-glass (SG) models are important examples of such richness [1-3]. Furthermore, the combination of these two highly nontrivial manifestations of disorder leads to a fascinating area of research in spin systems, which is not only a theoretical possibility. Actually, they can be found in diluted Ising-like antiferromagnets, like $\mathrm{Fe}_{x} \mathrm{Zn}_{1-x} \mathrm{~F}_{2}$ and $\mathrm{Fe}_{x} \mathrm{Mg}_{1-x} \mathrm{Cl}_{2}$ [4]. Additionally, recent investigations have suggested the diluted Ising-like dipolar ferromagnetic compound $\mathrm{LiHo}_{x} \mathrm{Y}_{1-x} \mathrm{~F}_{4}$ as a new candidate presenting these two types of disorder, bringing novel interesting and controversial issues [5-13]. For instance, the $\mathrm{LiHo}_{0.167} \mathrm{Y}_{0.833} \mathrm{~F}_{4}$ compound, in the absence of an applied transverse field $B_{t}$, displays $\chi_{3}$ (the lowest term of the nonlinear susceptibility $\chi_{n l}$ ) with a sharp peak at the freezing temperature $T_{f}$, which resembles a conventional second-order SG phase transition [14-16]. On the other hand, the sharp peak of $\chi_{3}$ becomes increasingly rounded when $B_{t}$ is enhanced, being located at the temperature $T^{*}$, which is lower than $T_{f}$ obtained in the absence of $B_{t}$ [17].

The suggestion that an effective longitudinal RF can be induced by the interplay of a transverse applied field

\footnotetext{
*carlosavjr@gmail.com
}

$B_{t}$ with the off-diagonal terms of the dipolar interactions in the $\mathrm{LiHo}_{x} \mathrm{Y}_{1-x} \mathrm{~F}_{4}$ [5-8] brought a new push to clarify the controversies of the experimental behavior of $\chi_{3}$ and, therefore, the meaning of $T^{*}$, i.e., whether or not it is a true SG transition temperature. In the droplet picture used in Refs. [5] and [6], the presence of a RF $h_{i}\left(B_{t}\right)$, induced by the uniform transverse field, suppresses the SG transition for the same reason that a uniform field does it in that picture [18]. On the other hand, within the mean-field Parisi's framework [19,20], Tabei and collaborators [7], using a quantum version of the Sherrington-Kirkpatrick (SK) model [21] with additional offdiagonal interactions, longitudinal $\mathrm{RF} h_{i}\left(B_{t}\right)$, and a transverse field $\Gamma\left(B_{t}\right)$, succeeded in reproducing the $\chi_{3}$ experimental behavior. Indeed, this result is a strong evidence that the $\mathrm{RF}$ plays an important role in the $\mathrm{LiHo}_{x} \mathrm{Y}_{1-x} \mathrm{~F}_{4}$ compound. These authors also suggested that the SG quantum criticality is unlikely in this transverse field, induced longitudinal RF scenario; additionally, susceptibility measurements presented evidence of a canonical SG behavior $[10,11]$. From that point of view, one can raise the question of what happens with the SG criticality in a regime where thermal fluctuations should be dominant as compared with the quantum ones. One possible consequence of the transverse field, induced longitudinal RF, is that the Almeida-Thouless (AT) line [22] can be suppressed, as suggested by numerical simulations in short-range-interaction SGs [23]. However, previous studies using a mean-field Parisi's framework have shown that the SK model with a RF does preserve the AT line [24-28]. Consequently, assuming that Parisi's mean-field theory is a 
valid framework to describe the SG problem with a transverse field, induced longitudinal RF, one can also raise the question of how the behavior of $\chi_{3}$ can be related with the AT line when a RF is present in the SK model. One can expect that the answer to this question may also help to clarify the meaning of the temperature $T^{*}$.

Therefore, in this work we present a detailed investigation of the role of a RF in the behavior of $\chi_{3}$ and its relation to the AT line in the SK model within the mean-field Parisi's framework. In order to relate with experimental verifications on the $\mathrm{LiHo}_{x} \mathrm{Y}_{1-x} \mathrm{~F}_{4}$ compound, we assume that $B_{t}$ is sufficiently small to assure that quantum fluctuations are negligible but enough to guarantee that the effective field-induced $\mathrm{RF} h_{i}\left(B_{t}\right)$ is still appreciable. When $h_{i}=0\left(B_{t}=0\right)$, it is known that $\chi_{3}$ is related with the SG susceptibility,

$$
\chi_{\mathrm{SG}}=(\beta / N) \sum_{i, j}\left[\left(\left\langle S_{i} S_{j}\right\rangle-\left\langle S_{i}\right\rangle\left\langle S_{j}\right\rangle\right)^{2}\right]_{\mathrm{av}},
$$

where, as usual, $\langle.$.$\rangle and [. .]_{\mathrm{av}}$ denote, respectively, thermal averages and an average over the disorder. The SG susceptibility has a term inversely proportional to the AT eigenvalue $\lambda_{\mathrm{AT}}$, the so-called replicon [29]. Therefore, the diverging behavior, $\chi_{3} \propto\left(T-T_{f}\right)^{-\gamma}$, in the SG transition is directly related with $\lambda_{\mathrm{AT}}=0$ at $T_{f}$, corresponding to the onset of replica symmetry breaking (RSB).

However, the situation changes considerably when $h_{i} \neq 0$ (i.e., $B_{t} \neq 0$ ). For instance, the RF can induce directly the SG order parameter, but only in the replica-symmetric (RS) approximation, since the RF is unable to produce any RSB. This result was demonstrated not only for infinite-ranged spin interactions [30], but also for the Bethe lattice [31]. As a consequence, the smooth behavior of the SG RS order parameter $q$ is not appropriate for identifying a SG transition of the SK model in the presence of a random field; however, such a transition may be related with the onset of RSB, associated with the replicon $\lambda_{\mathrm{AT}}=0$ [24]. In spite of this, the derivative of $q$ with respect to the temperature increases as one approaches $T_{f}$ from above; such an increase is responsible for the rounded maximum in $\chi_{3}$ at a temperature $T^{*}$, which does not coincide with the SG transition temperature $T_{f}$ (i.e., $T^{*}>T_{f}$ for a given applied random field). Thus, the maximum value of $\chi_{3}$ at $T^{*}$ should reflect the effects of the RF inside the paramagnetic (PM) phase instead of the nontrivial ergodicity breaking of the SG phase transition. Our results also suggest that $\chi_{3}$ still maintains a dependence on the replicon $\lambda_{\mathrm{AT}}$, although in a much more complicated way as compared with the case without the RF.

This paper is structured as follows. In the next section we define the model and the analytical procedure to be used; then, we calculate $\lambda_{\mathrm{AT}}$, the order parameters within the one-step replica-symmetry-breaking (1S-RSB) scheme, the susceptibilities $\chi_{1}, \chi_{3}$, as well as the temperature $T^{*}$ in the presence of RFs, following both Gaussian and bimodal distributions. In Sec. III we discuss the numerical solutions of the saddle-point equations for the order parameters and susceptibilities. Finally, the last section is reserved to the conclusions.

\section{MODEL AND SUSCEPTIBILITIES}

Herein we consider the infinite-range-interaction spin-glass model, defined by the following Hamiltonian:

$$
H=-\sum_{(i, j)} J_{i j} S_{i} S_{j}-\sum_{i=1}^{N} h_{i} S_{i}-H_{l} \sum_{i=1}^{N} S_{i},
$$

where $S_{i}= \pm 1, H_{l}$ represents a uniform field, and the sum $\sum_{(i, j)}$ applies to all distinct pairs of spins. The spin-spin couplings $\left\{J_{i j}\right\}$ and the magnetic random fields $\left\{h_{i}\right\}$ follow independent Gaussian probability distributions,

$$
P(X)=\left[\frac{1}{2 \pi \sigma^{2}}\right]^{1 / 2} \exp \left[-\frac{X^{2}-C}{2 \sigma^{2}}\right],
$$

where $X$ may represent either couplings or random fields; in the former case one has $\sigma=J / \sqrt{N}$ and $C=J_{0} / N$, whereas in the later, $\sigma=\Delta$ and $c=0$. We also consider a bimodal probability distribution,

$$
P\left(h_{i}\right)=p \delta\left(h_{i}-h_{0}\right)+(1-p) \delta\left(h_{i}+h_{0}\right),
$$

for the random fields $\left\{h_{i}\right\}$. We follow closely the procedure used in Ref. [24] to obtain the average free energy per spin, $f=-1 /(\beta N)\left[\ln Z\left(\left\{J_{i j}\right\},\left\{h_{i}\right\}\right)\right]_{J, h}$, where $Z\left(\left\{J_{i j}\right\},\left\{h_{i}\right\}\right)$ represents the partition function for a given quenched distribution of random couplings and fields; moreover, $[. .]_{J, h}$ denotes averages over these types of disorder, and $\beta=1 / T$. As usual, the replica method $[1,2,29]$ is applied; thus,

$$
-\beta f=\lim _{N \rightarrow \infty} \lim _{n \rightarrow 0} \frac{1}{N n}\left(\left[Z\left(\left\{J_{i j}\right\},\left\{h_{i}\right\}\right)^{n}\right]_{J, h}-1\right),
$$

where $Z^{n}$ corresponds to the replicated partition function. In the replica space, the average over the disorder may be evaluated and we adopt the $1 \mathrm{~S}-\mathrm{RSB}$; this procedure leads to the following free energy [26]:

$$
\begin{aligned}
\beta f= & \frac{(\beta J)^{2}}{4} x\left(q_{1}^{2}-q_{0}^{2}\right)-\frac{(\beta J)^{2}}{4}\left(1-q_{1}\right)^{2}+\frac{\beta J_{0}}{2} m^{2} \\
& -\frac{1}{x} \int D z \ln \int D v[2 \cosh \Xi(z, v)]^{x},
\end{aligned}
$$

with $D z \equiv \frac{1}{\sqrt{2 \pi}} d z \exp \left(-z^{2} / 2\right)$ and all integrals should be considered over the whole interval (from $-\infty$ to $+\infty$ ). The 1S-RSB internal field $\Xi(z, v)$ is given by

$$
\begin{aligned}
\Xi(z, v)= & \beta J\left(\sqrt{q_{0}+\Theta(1-n)} z+\sqrt{q_{1}-q_{0}} v\right) \\
& +\beta J_{0} m+\beta\left(H_{l}+n \Theta\right) .
\end{aligned}
$$

The equations above apply to both types of random fields, through the identifications $n=0$ and $\Theta=(\Delta / J)^{2}$ (Gaussian RF), whereas $n=1$ and $\Theta=h_{0}$ (bimodal RF). It should be mentioned that in the present work the results for the bimodal RF become independent of $p$, which may be seen by means of a change of variables $z \rightarrow-z$ [27]. Therefore, the analysis of the bimodal distribution becomes completely equivalent to that of a shifted uniform field given by $H_{l}+h_{0}$. Since the analysis of the SK model in the presence of a uniform field has been carried in the literature by many authors [26,27,32,33], from now on we focus our analysis to the Gaussian random 
field, for which the internal field of Eq. (7) becomes

$$
\begin{aligned}
\Xi(z, v)= & \beta J\left(\sqrt{q_{0}+(\Delta / J)^{2}} z+\sqrt{q_{1}-q_{0}} v\right) \\
& +\beta J_{0} m+\beta H_{l} .
\end{aligned}
$$

The 1S-RSB parameters $q_{0}, q_{1}$, and $x$ should extremize the free energy of Eq. (6), from which the RS solution is recovered when $q=q_{0}=q_{1}[32,33]$. The linear susceptibility $\chi_{1}=\left.\frac{\partial m}{\partial H_{l}}\right|_{H_{l} \rightarrow 0}$ is given by $\chi_{1}=\beta\left[1-q_{1}+x\left(q_{1}-q_{0}\right)\right]$ [20] when $J_{0}=0$. The nonlinear susceptibility $\chi_{3}$ can be obtained from $\chi_{3}=-\left.\frac{1}{3 !} \frac{\partial^{3} m}{\partial H_{l}^{3}}\right|_{H_{l} \rightarrow 0}$. Moreover, important effects on $\chi_{3}$ appear already inside the region where the RS solution is stable, more precisely, $\chi_{3}$ presents a rounded maximum at a temperature $T^{*}$, above the SG transition. Particularly, we can expand $q$ and $m$ in powers of $H_{l}$, for $J_{0}=0$, as (following Wada [14])

$$
\begin{gathered}
q\left(H_{l}\right)=Q_{0}+Q_{2} H_{l}^{2}, \\
m\left(H_{l}\right)=\chi_{1} H_{l}+\chi_{3} H_{l}^{3},
\end{gathered}
$$

which results in

$$
\chi_{3}(T)=\frac{\beta^{3}}{3}\left(3 J^{2} Q_{2}+1\right) I_{0},
$$

with $Q_{0}=\int D z \tanh ^{2} \Xi_{0}(z)$ and $Q_{2}=\left.\frac{\partial^{2} q}{\partial H_{l}^{2}}\right|_{H_{l} \rightarrow 0}$, where the RS internal field is obtained from Eq. (8) by setting $H_{l}=0$ and $q_{0}=q_{1}=q$, i.e., $\Xi_{0}(z)=\beta J\left(\sqrt{q+(\Delta / J)^{2}} z\right)$ and

$$
I_{0}=\int D z\left[\operatorname{sech}^{4} \Xi_{0}(z)-2 \operatorname{sech}^{2} \Xi_{0}(z) \tanh ^{2} \Xi_{0}(z)\right] .
$$

Moreover, $Q_{2}$ can be obtained as

$$
Q_{2}=\left.\frac{1}{2 !} \frac{\partial^{2} q}{\partial H_{l}^{2}}\right|_{H_{l} \rightarrow 0}=\frac{\beta^{2} I_{0}}{1-(\beta J)^{2} I_{0}},
$$

so that $\chi_{3}(T)$ becomes

$$
\chi_{3}(T)=-\frac{\beta^{3}}{3}\left[\frac{3(\beta J)^{2} I_{0}}{1-(\beta J)^{2} I_{0}}+1\right] I_{0} .
$$

These results hold when the RS solution is stable, given by a positive value of the eigenvalue $\lambda_{\mathrm{AT}}$ [24-28],

$$
\lambda_{\mathrm{AT}}=1-(\beta J)^{2} \int D z \operatorname{sech}^{4} \Xi_{0}(z) .
$$

Particularly, $\chi_{3}(T)$ can be written in terms of $\lambda_{\mathrm{AT}}$,

$$
\chi_{3}(T)=\frac{\beta^{3}}{3}\left[\frac{3}{\lambda_{\mathrm{AT}}+(\beta J)^{2} I_{1}}-2\right] I_{0},
$$

where

$$
I_{1}=2 \int D z \operatorname{sech}^{2} \Xi_{0}(z) \tanh ^{2} \Xi_{0}(z) .
$$

In the absence of RFs, $I_{1}=0$ in the PM phase, implying on a divergence of $\chi_{3}$ when $\lambda_{\mathrm{AT}}=0$, as expected [29]. Moreover, in the presence of RFs, one has that $I_{1}>0$, so that Eq. (16) leads to a rounded maximum at a temperature $T^{*}$.

\section{RESULTS AND DISCUSSION}

Numerical results are now presented. The effects of RFs on the SG order parameters $q_{0}, q_{1}, \delta \equiv q_{1}-q_{0}$, susceptibilities $\chi_{1}$ and $\chi_{3}$, as well as the stability of the RS solution (i.e., $\lambda_{\mathrm{AT}}$ ) are discussed. In particular, the onset of RSB (location of $T_{f}$ ) and how $\chi_{3}$ behaves in the neighborhood of the SG phase transition are studied.

For instance, Fig. 1 shows that the SG order-parameter behavior, signaling $\operatorname{RSB}(\delta>0)$, occurs at lower temperatures due to the presence of RFs, i.e., the increase of $\Delta / J$ moves $T_{f}$ to lower temperatures. The freezing temperature $T_{f}$, which is located within the 1S-RSB scheme as the onset of the parameter $\delta$, is shown herein to coincide with $\lambda_{\mathrm{AT}}=0$. As presented in the inset of Fig. 1, the RFs induce the order parameters $q_{0}$ and $q_{1}$ for $T>T_{f}$, where the RS solution is stable $\left[q=q_{0}=q_{1}, \delta=0\right.$ and $\left.\lambda_{\mathrm{AT}}>0\right]$, characterizing the PM phase. In the cases $(\Delta / J)>0$ one notices that $T^{*}>T_{f}$, with the arrows indicating the temperature $T^{*}$ where $\chi_{3}$ presents a rounded maximum.

As shown in Fig. 2, the magnetic susceptibility $\chi_{1}$ exhibits a clear cusp at $T_{f}$ in the absence of the RF, whereas in the presence of a RF, one notices a smooth behavior around $T_{f}$. Below this temperature, the 1S-RSB and RS solutions become distinct, with the former presenting higher values, being weakly dependent on the temperature.

In Fig. 3 we present results for the nonlinear susceptibility $\chi_{3}$, computed directly from the numerical derivatives $\chi_{3}=$ $-\left.\frac{1}{3 !} \frac{\partial^{3} m}{\partial H_{l}^{3}}\right|_{H_{l} \rightarrow 0}$. As a check, for $T \geqslant T_{f}$, we verified that these results coincide with those obtained from Eq. (16). For the case without RFs, $\chi_{3}$ shows a strong divergence at $T_{f}$ (see inset in Fig. 3); however, the presence of a RF eliminates this

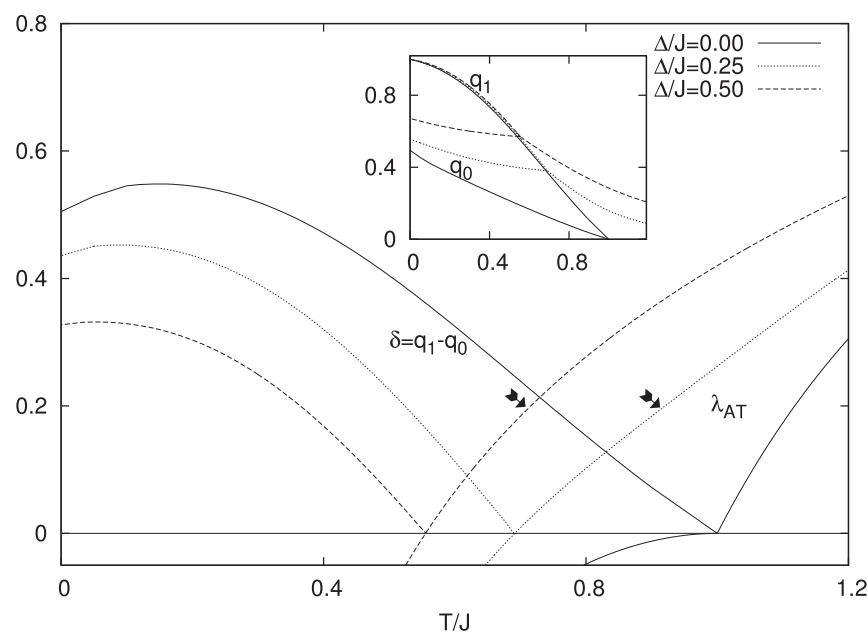

FIG. 1. The 1S-RSB parameter $\delta \equiv q_{1}-q_{0}$ and the eigenvalue $\lambda_{\mathrm{AT}}$ are presented versus the dimensionless temperature $T / J$, for typical values of $\Delta / J$. The inset shows the SG parameters $q_{1}$ and $q_{0}$ separately versus the dimensionless temperature. The freezing temperature $T_{f}$ is identified with the onset of RSB, where $\lambda_{\mathrm{AT}}=0$, or equivalently, where the parameter $\delta$ becomes nonzero. The arrows indicate the temperature $T^{*}$, where $\chi_{3}$ presents a rounded maximum, showing that $T^{*}>T_{f}$. Due to the usual numerical difficulties, the low-temperature results [typically $(T / J)<0.05)$ ] correspond to smooth extrapolations from higher-temperature data. 


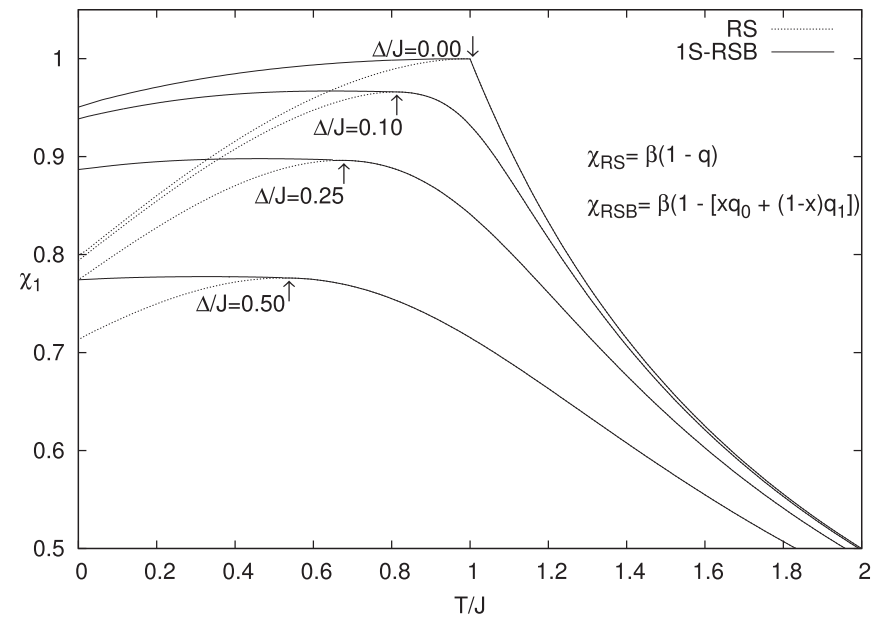

FIG. 2. Magnetic susceptibility $\chi_{1}$ versus $T / J$ for different values of $\Delta / J$. The arrows indicate the onset of the RSB solution $\left(\lambda_{\mathrm{AT}}=\right.$ 0 ), defining the temperature $T_{f}$. Below $T_{f}$, solid and dotted lines indicate linear susceptibilities computed using $1 \mathrm{~S}-\mathrm{RSB}\left(\chi_{\mathrm{RSB}}\right)$ and RS $\left(\chi_{\mathrm{RS}}\right)$ solutions, respectively. Due to the usual numerical difficulties, the low-temperature results [typically $(T / J)<0.05$ ] correspond to smooth extrapolations from higher-temperature data.

divergence, and rounded maxima appear in the $\chi_{3}$ curves, defining the temperature $T^{*}$ for each value of $\Delta / J$. It is important to remark that $T^{*}$ is always higher than $T_{f}$. Furthermore, the $T^{*}$ and $\chi_{3}$ values decrease for increasing values of $\Delta / J$. Within the RSB region, similarly to what was shown for the linear susceptibility $\chi_{1}$ (cf. Fig. 2), $\chi_{3}$ also presents a split between the results with RS and 1S-RSB solutions. However, differently from $\chi_{1}$, the nonlinear susceptibility $\chi_{3}$ displays an evident discontinuity at $T_{f}$ when the $1 \mathrm{~S}-\mathrm{RSB}$ solution is adopted.

An important quantity in Eq. (16) is the denominator, $\gamma=$ $\lambda_{\mathrm{AT}}+(\beta J)^{2} I_{1}$, which is illustrated in detail in Fig. 4 versus

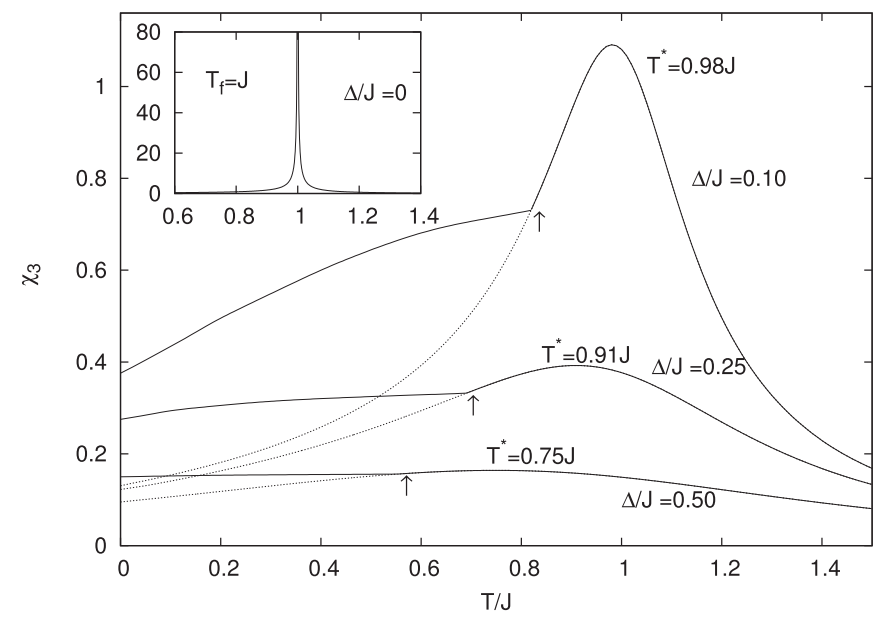

FIG. 3. The susceptibility $\chi_{3}$ as a function of $T / J$ for different values of $\Delta / J$. The arrows indicate the onset of the RSB solution $\left(\lambda_{\text {AT }}=0\right)$, defining the temperature $T_{f}$. Below $T_{f}$, solid and dotted lines indicate $1 \mathrm{~S}-\mathrm{RSB}$ and RS solutions, respectively. The temperature $T^{*}$, where $\chi_{3}$ presents a rounded maximum, is estimated in each case shown. In the inset we exhibit the $\chi_{3}$ behavior without the RF.

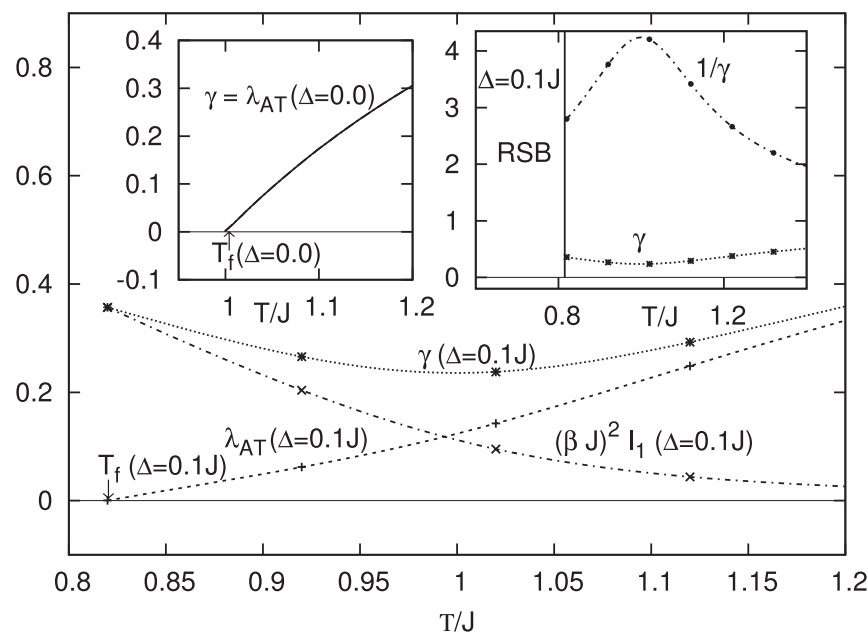

FIG. 4. The quantities appearing in the denominator of Eq. (16), $\gamma=\lambda_{\mathrm{AT}}+(\beta J)^{2} I_{1}$, are presented versus $T / J$ for $(\Delta / J)=0.1$. The arrows locate the freezing temperature $T_{f}$. The inset on the right shows in detail the behaviors of $\gamma$ and $1 / \gamma$, for $(\Delta / J)=0.1$, in the region where the RS solution is stable (to the left of this region one should use RSB); the quantity $1 / \gamma$ presents a rounded maximum, which is directly related with that found in $\chi_{3}$. The inset on the left shows in detail the behaviors of $\gamma$ and $\lambda_{\mathrm{AT}}$, for $\Delta / J=0.0$, which are responsible for the divergence of $\chi_{3}$ in the absence of RFs.

$T / J$ for the typical value $(\Delta / J)=0.1$. As a comparison, the inset on the left shows the behavior of $\gamma$ and $\lambda_{\mathrm{AT}}$, for $(\Delta / J)=0.0$; in this case, $\gamma=0$ leads to the divergence of $\chi_{3}$ in the absence of RFs. When $(\Delta / J)>0$, one has that the contribution $(\beta J)^{2} I_{1}>0$, so that now $\gamma>0$. The two contributions, $\lambda_{\mathrm{AT}}$ (that increases for increasing values of $T / J$ ) and $(\beta J)^{2} I_{1}$ (that decreases for increasing values of $T / J$ ), are presented separately, leading to a minimum value for $\gamma$, which is found to occur very close to the temperature $T^{*}$. The inset on the right shows the maximum attained by $1 / \gamma$, appearing inside the region where RS is stable; to the left of this region, one should analyze these quantities within $\mathrm{RSB}$. This maximum is directly related with the one presented in Fig. 3, at the temperature $T^{*}$, and since this temperature is found in the $\mathrm{RS}$ region, we consider the rounded maximum to occur in the paramagnetic phase. One should remember the role played by the RF on the replicon, leading to a shift in the freezing temperature towards lower temperatures, i.e., $T_{f}(\Delta>0)<$ $T_{f}(\Delta=0)[24,26,27]$. Hence, in Fig. 4 one notices that in the temperature range $T_{f}(\Delta>0)<T<T_{f}(\Delta=0)$ the behavior of the denominator $\gamma$ changes completely from decreasing to increasing. This inversion yields the minimum of $\gamma$, which is the ultimate mechanism leading to the rounded maximum of $\chi_{3}$ at $T^{*}$.

In Fig. 5 we present the phase diagram of the model, showing the paramagnetic and SG phases. The SG phase is associated with the onset of RSB, being signaled by the zero of the replicon of Eq. (15) (i.e., $\lambda_{\mathrm{AT}}=0$ ), which defines the freezing temperature $T_{f}$. The temperature $T_{f}$ is lowered due to the RFs; in fact, such a decrease in $T_{f}$ can be verified analytically for $(\Delta / J) \ll 1$, in which case an expansion can 


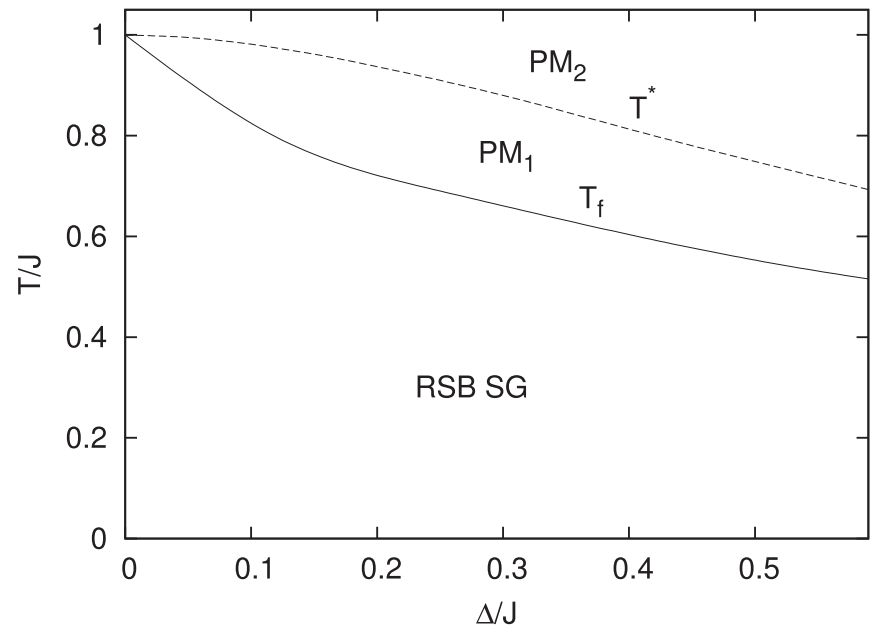

FIG. 5. Phase diagram $T / J$ versus $\Delta / J$ showing the paramagnetic and SG phases. The freezing temperature $T_{f}$, signaling the onset of RSB, defines the SG phase for $T<T_{f}$. For completeness, we also present the line associated with the maximum of $\chi_{3}$, defining the temperature $T^{*}$ (dashed line). The possibility of two paramagnetic phases $\left(\mathrm{PM}_{1}\right.$ and $\left.\mathrm{PM}_{2}\right)$ is discussed in the text.

be obtained from Eq. (15) [24,27]:

$$
\frac{T_{f}}{J} \approx 1-\left(\frac{3}{4}\right)^{1 / 3}\left(\frac{\Delta}{J}\right)^{2 / 3}
$$

The dashed line in Fig. 5 represents the temperature $T^{*}$ $\left(T^{*}>T_{f}\right.$ ), characterizing the maximum of $\chi_{3}$, which exists for any $(\Delta / J)>0$. For $T>T^{*}$ the phase $\mathrm{PM}_{2}$ occurs, along which one has weak correlations and consequently, the usual paramagnetic type of behavior. However, close to $T^{*}$, and particularly for temperatures in the range $T_{f}<T<T^{*}$, one expects a rather nontrivial behavior in real systems, as happens with the compound $\mathrm{LiHo}_{x} \mathrm{Y}_{1-x} \mathrm{~F}_{4}$, resulting in very controversial interpretations [5-11]. Due to such aspects, herein we call the temperature region $T_{f}<T<T^{*}$ of $\mathrm{PM}_{1}$. The line $\mathrm{PM}_{1}-\mathrm{PM}_{2}$ may not characterize a real phase transition, in the sense of a diverging $\chi_{3}$, but the region $\mathrm{PM}_{1}$ is certainly characterized by a rather nontrivial dynamics. As shown in Fig. 4, the region $\mathrm{PM}_{2}$ presents small values for the quantity $I_{1}$ [cf. the denominator of Eq. (16)], whereas along $\mathrm{PM}_{1}$ the couplings between RFs and spins become dominant, as compared with thermal fluctuations, and $I_{1}$ increases significantly. As a possible relation, one should have a growth of free-energy barriers in the region $\mathrm{PM}_{1}$, leading to a slow dynamics, whereas only below $T_{f}$ does the nontrivial ergodicity breaking appear, typical of RSB in SG systems. It is important to remember also that Griffiths singularities are found currently in disordered magnetic systems, like for site-diluted ferromagnets [34], as well for a ferromagnet in a random field [35]. Whether the region $\mathrm{PM}_{1}$ in the present problem may be related to this latter type of behavior is a matter for further investigation.

As already mentioned and addressed in several works [14-16], in the absence of RFs, the SG phase transition is given by the divergence of $\chi_{3}$ at $T_{f}$. In Parisi's mean-field theory this divergence is directly related with the onset of RSB, signaled by a zero of the replicon $\lambda_{\mathrm{AT}}=0$ [29]. However, the presence of RFs induces the SG order parameter $q$ in the PM phase, within the RS solution. Moreover, $\chi_{3}$ no longer diverges at the SG transition temperature but instead, presents a rounded maximum at $T^{*}$, which becomes smoother as $\Delta / J$ increases. Such a difference with respect to the case without RFs can be understood from Eqs. (16) and (17). In fact, the term $I_{1}$ in Eq. (16), which is responsible for these effects, can be rewritten as

$$
I_{1}=2(q-r)
$$

with

$$
\left\langle S^{\alpha} S^{\beta}\right\rangle \equiv q=\int D z \tanh ^{2} \Xi_{0}(z),
$$

and

$$
\left\langle S^{\alpha} S^{\beta} S^{\gamma} S^{\delta}\right\rangle \equiv r=\int D z \tanh ^{4} \Xi_{0}(z) .
$$

These equations lead to $\gamma=\lambda_{\mathrm{AT}}+(\beta J)^{2} I_{1}=1-(\beta J)^{2}(1-$ $4 q+3 r)$, which is precisely the longitudinal eigenvalue of the RS stability analysis [22,29]. This longitudinal eigenvalue is related with the magnitude of the fluctuations of the of RS SG order parameter $q$. Hence, the maximum of $\chi_{3}$ at $T^{*}$ becomes completely unrelated with the SG phase transition when $\Delta$ departs from zero, being directly associated with the longitudinal eigenvalue.

\section{CONCLUSIONS}

The role of random fields on the spin-glass freezing temperature, as well as on the nonlinear susceptibility, was analyzed. For that, we have investigated the Sherrington-Kirkpatrick model in the presence of random fields, following a Gaussian distribution characterized by a width $\Delta$, within a one-step replica-symmetry-breaking procedure. We have shown that the divergence in $\chi_{3}$ only occurs in the absence of random fields and that $\chi_{3}$ exhibits a broad maximum at a temperature $T^{*}$ for $\Delta>0$. The freezing temperature $T_{f}$ is associated with the onset of replica symmetry breaking, signaled by the zero of the Almeida-Thouless (replicon) eigenvalue, occurring at lower temperatures, i.e., $T^{*}>T_{f}$ for a given value of $\Delta$.

The splitting between $T_{f}$ and $T^{*}$, for $\Delta>0$, was studied by analyzing the contribution due to the random fields in the replica-symmetry spin-glass order parameter. Particularly, we have shown that the behavior of $\chi_{3}$ is not regulated only by the spin-spin correlations associated to the Almeida-Thouless line, but also to correlations coming from the longitudinal eigenvalue. These correlations play an important role inside the paramagnetic phase, when the random fields are applied, being responsible for the maximum in $\chi_{3}$, although they are not directly associated with the spin-glass phase transition.

Although the present results refer a specific model, we expect they could shed some light in the theoretical and experimental description of disordered magnetic systems like, for instance, the compound $\mathrm{LiHo}_{x} \mathrm{Y}_{1-x} \mathrm{~F}_{4}$. Considering recent observations in $\mathrm{LiHo}_{x} \mathrm{Y}_{1-x} \mathrm{~F}_{4}$, we follow the proposal that an applied transverse field $B_{t}$ induces longitudinal random fields [7], and thus, we assume herein $\Delta=\Delta\left(B_{t}\right)$. In this way, one can interpret the present results, e.g., the temperatures $T^{*}$ and $T_{f}$, as manifestations of the transverse field. Based 
on this, we point out below two possibilities which may contribute to elucidate the recent controversies on this system [5-11]: (i) The temperature $T^{*}$ associated with the rounded maximum in the nonlinear susceptibility does not signal any phase transition, being an effect of random fields inside the paramagnetic phase, although it is related to a minimum of the longitudinal eigenvalue, and hence, to large fluctuations in the replica-symmetric spin-glass order parameter. A true spin-glass phase transition, indicated through the SherringtonKirkpatrick model, by means of the Almeida-Thouless line, should occur at the lower temperature $T_{f}$. (ii) There is no spin-glass phase transition in $\mathrm{LiHo}_{x} \mathrm{Y}_{1-x} \mathrm{~F}_{4}$ in the presence of a transverse field, implying that the replica-symmetry-breaking procedure does not apply to this compound. Certainly, these two points require meticulous experimental observations for temperatures around $T^{*}$, which has been the most investigated temperature region so far, as well as below $T^{*}$, representing a challenge for experiments.

\section{ACKNOWLEDGMENTS}

This work was supported by the Brazilian funding agencies CNPq, through Projects No. 306720/2013-2, No. 474559/2013-0, and No. 443565/2014-7, and CAPES.
[1] V. Dotsenko, 2001 Introduction to the Replica Theory of Disordered Statistical Systems (Cambridge University Press, Cambridge, UK, 2001).

[2] H. Nishimori, Statistical Physics of Spin Glasses and Information Processing (Oxford University Press, Oxford, UK, 2001).

[3] 1998 Spin Glasses and Random Fields, edited by A. P. Young (World Scientific, Singapore, 1998).

[4] D. P. Belanger, in Spin Glasses and Random Fields, edited by A. P. Young (World Scientific, Singapore, 1998), p. 251.

[5] M. Schechter and P. C. E. Stamp, Phys. Rev. Lett. 95, 267208 (2005).

[6] M. Schechter and N. Laflorencie, Phys. Rev. Lett. 97, 137204 (2006).

[7] S. M. A. Tabei, M. J. P. Gingras, Y.-J. Kao, P. Stasiak, and J.-Y. Fortin, Phys. Rev. Lett. 97, 237203 (2006).

[8] M. Gingras and P. Henelius, J. Phys: Conf. Ser. 320, 012001 (2011).

[9] P. E. Jönsson, R. Mathieu, W. Wernsdorfer, A. M. Tkachuk, and B. Barbara, Phys. Rev. Lett. 98, 256403 (2007).

[10] C. Ancona-Torres, D. M. Silevitch, G. Aeppli, and T. F. Rosenbaum, Phys. Rev. Lett. 101, 057201 (2008).

[11] J. A. Mydosh, Rep. Prog. Phys. 78, 052501 (2015).

[12] J. F. Fernandez, Phys. Rev. B 82, 144436 (2010).

[13] J. J. Alonso, Phys. Rev. B 91, 094406 (2015).

[14] K. Wada and H. Takayama, Prog. Theor. Phys. 64, 327 (1980).

[15] M. Suzuki, Prog. Theor. Phys. 58, 1151 (1977).

[16] J. Chalupa, Solid State Commun. 22, 315 (1977).

[17] W. Wu, D. Bitko, T. F. Rosenbaum, and G. Aeppli, Phys. Rev. Lett. 71, 1919 (1993).
[18] D. S. Fisher and D. A. Huse, Phys. Rev. Lett. 56, 1601 (1986).

[19] G. Parisi, J. Phys. A 13, 1101 (1980).

[20] G. Parisi, J. Phys. A 13, 1887 (1980).

[21] D. Sherrington and S. Kirkpatrick, Phys. Rev. Lett. 35, 1792 (1975).

[22] J. R. L. de Almeida and D. J. Thouless, J. Phys. A 11, 983 (1978).

[23] A. P Young and H. G. Katzgraber, Phys. Rev. Lett. 93, 207203 (2004).

[24] R. F. Soares, F. D. Nobre, and J. R. L. de Almeida, Phys. Rev. B 50, 6151 (1994).

[25] E. Nogueira, F. D. Nobre, F. A. da Costa, and S. Coutinho, Phys. Rev. E 57, 5079 (1998); 60, 2429(E) (1999).

[26] S. G. Magalhães, C. V. Morais, and F. D. Nobre, J. Stat. Mech. (2011) P07014.

[27] C. V. Morais, S. G. Magalhães, and F. D. Nobre, J. Stat. Mech. (2012) P01013.

[28] J. M. de Araújo, F. D. Nobre, and F. A. da Costa, Phys. Rev. E 61, 2232 (2000).

[29] K. Binder and A. P. Young, Rev. Mod. Phys. 58, 801 (1986).

[30] T. Schneider and E. Pytte, Phys. Rev. B 15, 1519 (1977).

[31] F. Krzakala, F. Ricci-Tersenghi, and L. Zdeborová, Phys. Rev. Lett. 104, 207208 (2010)

[32] R. Pirc, B. Tadić, and R. Blinc, Phys. Rev. B 36, 8607 (1987).

[33] D.-H. Kim and J.-J. Kim, Phys. Rev. B 66, 054432 (2002).

[34] R. B. Griffiths, Phys. Rev. Lett. 23, 17 (1969).

[35] V. Dotsenko, J. Stat. Phys. 122, 197 (2006); J. Phys. A: Math Gen. 27, 3397 (1994). 